\title{
Prevalence of amyloid- $\beta$ pathology in distinct variants of primary progressive aphasia
}

David Bergeron $^{1,2}$, Maria L. Gorno-Tempini ${ }^{3}$, Gil D. Rabinovici ${ }^{3}$, Miguel A. Santos-Santos ${ }^{3,4,5}$, William Seeley $^{3}$, Bruce L. Miller ${ }^{3}$, Yolande Pijnenburg ${ }^{2}$, M. Antoinette Keulen ${ }^{2}$, Colin Groot ${ }^{2}$, Bart N.M. van Berckel $^{6}$, Wiesje M. van der Flier ${ }^{2}$, Philip Scheltens ${ }^{2}$, Jonathan D. Rohrer ${ }^{7}$, Jason D. Warren ${ }^{7}$, Jonathan M. Schott ${ }^{7}$, Nick C. Fox ${ }^{7}$, Raquel Sanchez-Valle ${ }^{8}$, Oriol Grau-Rivera ${ }^{8}$, Ellen Gelpi ${ }^{8,9}$, Harro Seelaar ${ }^{10}$, Janne M. Papma ${ }^{10}$, John C. van Swieten ${ }^{10}$, John R. Hodges ${ }^{11,12,13}$, Cristian E. Leyton ${ }^{11,13,14}$, Olivier Piguet ${ }^{11,12,13}$, Emily J. Rogalsky ${ }^{14,15}$, Marsel M. Mesulam ${ }^{15}$, Lejla Koric ${ }^{16}$, Kristensen Nora ${ }^{16}$, Jérémie Pariente ${ }^{17}$, Bradford Dickerson $^{18}$, Ian R. Mackenzie ${ }^{19}$, Ging-Yuek R. Hsiung ${ }^{19}$, Serge Belliard ${ }^{19}$, David J. Irwin ${ }^{20}$, David A. Wolk ${ }^{21}$, Murray Grossman ${ }^{21,22}$, Matthew Jones ${ }^{23,24}$, Jennifer Harris ${ }^{24}$, David Mann ${ }^{25}$, Julie S. Snowden $^{24}$, Patricio Chrem-Mendez ${ }^{26}$, Ismael L. Calandri ${ }^{26}$, Alejandra A. Amengual ${ }^{26}$, Carole MiguetAlfonsi ${ }^{27}$, Eloi Magnin ${ }^{27}$, Giuseppe Magnani ${ }^{28}$, Roberto Santangelo ${ }^{28}$, Vincent Deramecourt ${ }^{29}$, Florence Pasquier $^{29}$, Niklas Mattsson ${ }^{30}$, Christer Nilsson ${ }^{30}$, Oskar Hansson ${ }^{30,31}$, Julia Keith ${ }^{32}$, Mario Masellis ${ }^{33,34}$, Sandra E. Black ${ }^{33,34}$, Jordi A. Matías-Guiu ${ }^{35}$, María-Nieves Cabrera-Martin ${ }^{35}$, Claire Paquet ${ }^{36,37}$, Julien Dumuirger $^{36}$, Marc Teichmann ${ }^{38}$, Marie Sarazin ${ }^{39,40}$, Michel Bottlaender ${ }^{39,40}$, Bruno Dubois ${ }^{41}$, Christopher C. Rowe ${ }^{42,43}$, Victor L. Villemagne ${ }^{42,43}$, Rik Vandenberghe ${ }^{44}$, Elias Granadillo ${ }^{45,46}$, Edmond Teng ${ }^{47}$, Mario Mendez $^{48}$, Philipp T. Meyer ${ }^{49}$, Lars Frings ${ }^{49}$, Alberto Lleó ${ }^{50,51,52}$, Rafael Blesa ${ }^{50,51}$, Juan Fortea ${ }^{50,51}$, Sang Won Seo ${ }^{53}$, Janine Diehl-Schmid ${ }^{54}$, Timo Grimmer ${ }^{54}$, Kristian Steen Frederiksen ${ }^{55}$, Pascual Sánchez-Juan ${ }^{56}$, Gaël Chételat $^{57}$, Willemijn Jansen ${ }^{58,59}$, Rémi W. Bouchard ${ }^{1}$, Robert Jr. Laforce ${ }^{1,60}$, Pieter Jelle Visser ${ }^{5,58}$ \& Rik Ossenkoppele 2,30

1 Université Laval, Clinique Interdisiplinaire de Mémoire de l’Enfant-Jésus, Quebec, QC, Canada 2 VU University Medical Center, Alzheimer Center, Department of Neurology, Amsterdam, Netherlands

3 University of California San Fransisco, Memory \& Aging Center, Department of Neurology, San Fransisco, CA, USA

4 L'Hospitalet de Llobregat, Cognition and brain plasticity group [Bellvitge biomedical research instituteIDIBELL], Barcelona, Spain

5 L'Hospitalet de Llobregat, Fundació ACE. Institut Català de Neurociències Aplicades, UIC-Barcelona, Barcelona, ES

6 VU University Medical Center, Department of Radiology and Nuclaer Medicine, Amsterdam, Netherlands

7 University College London, Dementia Research Centre, UCL Institute of Neurology, London, UK 8 Institut d'investigacions Biomèdiques August Pi i Sunyer, Alzheimer's disease and other cognitive disorders unit, Barcelona, Spain

9 Medical University of Vienna, Institute of Neurology

Vienna, Austria

10 Erasmus MC - University Medical Center, Alzheimer center, Department of Neurology, Rotterdam, Netherlands

11 The University of Sydney, Brain and Mind Centre, School of Medical Sciences, Sydney, Australia

12 The University of New South Wales, Neuroscience Research Australia and School of Medical Sciences, Sydney, Australia

13 Australian Research Council Centre of Excellence in Cognition and its Disorders, Sydney, Australia

14 Rush University, Neurological Sciences, Chicago, IL, USA

15 Northwestern University Medical School, Cognitive Neurology and Alzheimer Disease Center, Chicago, IL, USA

16 Hopital de la Timone, Service de Neurologie et de Neuropsychologie, Marseille, France

This is the author manuscript accepted for publication and has undergone full peer review but has not been through the copyediting, typesetting, pagination and proofreading process, which may lead to differences between this version and the Version of Record. Please cite this article as doi: $10.1002 /$ ana.25333 
17 Université de Toulouse, Inserm, ToNIC, Toulouse NeuroImaging Center, Toulouse, France 18 Harvard Medical School, Frontotemporal Dementia Unit, Department of Neurology, Massachusetts

Alzheimer's Disease Research Center, Boston, MA, USA

19 University of British Columbia, Division of Neurology, Department of Medicine, Vancouver, Canada 20 University of Pennsylvania, Center for Neurodegenerative Disease Research, Department of Pathology and Laboratory Medicine, Philadelphia, PA, USA

21 University of Pennsylvania, Department of Neurology, Philadelphia, PA, USA

22 University of Pennsylvania, the Penn Frontotemporal Degeneration Center, Philadelphia, PA, USA

23 Greater Manchester Neurosciences Centre, Cerebral Function Unit, Manchester, UK

24 University of Manchester, School of Community-Based Medicine, Manchester, UK

25 University of Manchester, Division of Neuroscience and Experimental Psychology, School of Biological Sciences, Manchester, UK

26 Neurological Research Institute (FLENI), Center of Aging and Memory, Buenos Aires, Argentina

27 University of Bourgogne Franche-Comté (UBFC), Regional Memory Center (CMRR), Department of

Neurology, CHRU Besançon and Integrative and clinical Neurosciences Lab, Besançon, France

28 Vita-Salute University and IRCCS-San Raffaele Hospital, Department of Neurology, INSPE, Milan, Italy

29 Université Lille Nord de France, INSERM U1171, Lille, France

30 Lund University, Clinical Memory Research Unit, Department of Clinical Sciences, Lund, Sweden 31 Skåne University Hospital, Neuropsychiatric Clinic, Malmö, Sweden

32 Sunnybrook Health Sciences Centre, University of Toronto, Anatomical Pathology, Toronto, ON, Canada

33 Sunnybrook Health Sciences Centre, University of Toronto, Department of Medicine (Neurology), Toronto, ON, Canada

34 Sunnybrook Health Sciences Centre, University of Toronto, Hurvitz Brain Sciences Research Program, Toronto, ON, Canada

35 Hospital Clinico San Carlos, San Carlos Health Research Institute, Universidad Complutense, Department of Neurology and Nuclear Medicine, Madrid, Spain

36 Lariboisière-Fernand-Widal Hospital, Memory Center, Department of Neurology, Paris, France 37 Lariboisière-Fernand-Widal Hospital, Department of Pathology, Paris, France

38 Université Paris Descartes, Sorbonne Paris Cité, Centre Hospitalier Sainte Anne, Unit of Neurology of Memory and Language, Paris, France

39 Service Hospitalier Frederic Joliot, ERL 9218 CNRS, CEA, Orsay, Île-de-France, France

40 Universite Paris-Sud, IMIV, UMR 1023 Inserm, CEA, Orsay, Île-de-France, France

41 Hôpital Universitaire de la Pitié Salpêtrière, Centre des Maladies Cognitives et Comportementales, Paris, France

42 Austin Health, Department of Molecular Imaging \& Therapy, Melbourne, VIC, Australia

43 University of Melbourne, Department of Medicine, Melbourne, VIC, Australia

44 University Hospital Leuven, Department of Neurology, Leuven, Belgium

45 University of California Los Angeles, Department of Neurology, Los Angeles, CA, USA

46 VA Greater Los Angeles Healthcare System, Los Angeles, CA, USA

47 University of California Los Angeles, Department of Neurology, Neurobehavior Service, Los Angeles, CA, USA

48 West Los Angeles VA Medical Center, Neurobehavior Unit, Los Angeles, CA, USA

49 University Hospital of Freiburg, Department of Nuclear Medicine, Faculty of Medicine, Freiburg, Germany

50 Hospital de la Santa Creu i Sant Pau, Memory Unit, Department of Neurology, Barcelona, Spain

51 Universitat Autónoma de Barcelona, Biomedical Research Institute Sant Pau, Barcelona, Spain 
52 Centro de Investigacion Biomedica en Red sobre Enfermedades Neurodegenerativas, Madrid, Madrid, Spain

53 Samsung Medical Center, Sungkyunkwan University School of Medicine, Department of Neurology, Seoul, South-Korea

54 Technische Universität München, Department of Psychiatry and Psychotherapy, München, Germany 55 Danish Dementia Research Center, Department of Neurology, Copenhagen, Denmark 56 Universitary Hospital Marqués de Valdecilla, Neurology Service, Santander, Spain 57 Université de Caen-Normandie, Inserm UMR-S U1237, Caen, France

58 Maastricht University, Department of Psychiatry \& Neuropsychology, School for Mental Health and Neuroscience, Maastricht, Netherlands

59 Banner Alzheimer's Institute, Phoenix, AZ, USA

60 Université Laval, Faculté de Médecine, Département des Sciences Neurologiques, Quebec, QC, Canada 


\begin{abstract}
Objective: To estimate the prevalence of amyloid-positivity, defined by PET/CSF biomarkers and/or neuropathological examination, in primary progressive aphasia (PPA) variants.
\end{abstract}

Methods: We conducted a meta-analysis with individual participant data from 1,251 patients diagnosed with PPA (including logopenic [lvPPA, $n=443$ ], non-fluent [nfvPPA, $n=333$ ], semantic [svPPA, $n=401$ ] and mixed/unclassifiable [PPA-M/U, $n=74$ ] variants of PPA) from 36 centers, with a measure of amyloid- $\beta$ pathology (CSF [ $n=600]$ ), PET [n=366] and/or autopsy [n=378]) available. The estimated prevalence of amyloidpositivity according to PPA variant, age and apolipoprotein E (APOE) $\varepsilon 4$ status was determined using generalized estimating equation models.

Results: Amyloid- $\beta$ positivity was more prevalent in lvPPA (86\%) than in nfvPPA $(20 \%)$ or svPPA $(16 \%)(\mathrm{p}<0.001)$. Prevalence of amyloid- $\beta$ positivity increased with age in nfvPPA (from 10\% at age 50 to $27 \%$ at age $80, \mathrm{p}<0.01$ ) and svPPA (from $6 \%$ at age 50 to $32 \%$ at age $80, \mathrm{p}<0.001)$, but not in lvPPA $(\mathrm{p}=0.94)$. Across PPA variants, APOE $\varepsilon 4$ carriers were more often amyloid- $\beta$ positive (58.0\%) than non-carriers (35.0\%, $\mathrm{p}<0.001)$. Autopsy data revealed Alzheimer's disease (AD) pathology as the most common pathologic diagnosis in lvPPA (76\%), frontotemporal lobar degeneration - TDP-43 in svPPA (80\%) and frontotemporal lobar degeneration-TDP-43/tau in nfvPPA (64\%).

Interpretation: This study shows that the current PPA classification system helps to predict underlying pathology across different cohorts and clinical settings, and suggests that age and APOE genotype should be taken into account when interpreting $A \beta$ biomarkers in PPA patients.

This article is protected by copyright. All rights reserved. 


\section{Introduction}

Primary progressive aphasia (PPA) is a clinical syndrome characterized by progressive loss of language function in the setting of focal degeneration of the dominant-hemisphere language network (1). Although first described in late nineteenth century by Pick and Dejerine and Serieux, the notion of isolated, progressive aphasia in the context of a neurodegenerative condition only came to broader medical/scientific attention in 1982 with Dr. Mesulam's seminal observations (2). Since then, the nosology of PPA has been a field of intense investigation. The first official set of criteria for PPA defined two variants - 'progressive non-fluent aphasia' and 'semantic aphasia and associative agnosia' (3) - which were included under the rubric of fronto-temporo-lobar degeneration (FTLD). As a consequence, amyloid- $\beta$ (A $\beta$ ) pathology (a neuropathological hallmark of Alzheimer's disease [AD]) observed at autopsy in patients with PPA (4-6) was initially considered a co-morbid, age-related process (7-9). In 2004, cluster analyses of clinical and anatomical data brought Gorno-Tempini and colleagues to define a third ("logopenic”) variant of PPA (lvPPA), which was predicted to be primarily due to AD (10). The high prevalence of amyloid- $\beta$ positivity in lvPPA was confirmed using molecular imaging (11), contributing to its label as the "language variant of AD". However, other studies showed high prevalence of AD pathology at autopsy in progressive non-fluent aphasia and, to a lesser extent, in semantic dementia (12-17). Inconsistencies between the newly described variant and existing PPA criteria - for instance the overlap between the 1998 Neary criteria for progressive non-fluent aphasia (3) and the initial descriptions of logopenic aphasia (10) - became a growing source of confusion for clinicians and researchers.

To improve uniformity of case reporting and reliability of research results, a comprehensive set of consensus criteria for PPA was published in 2011 (18). Based on specific language profiles, three distinct variants were proposed: a non-fluent variant 
(nfvPPA), characterized by effortful speech output, agrammatism, and apraxia of speech, with relative sparing of single-word comprehension; a semantic variant (svPPA), distinguished by loss of word and object meaning, with fluent and grammatically correct speech; and a logopenic variant (lvPPA), defined by the co-occurrence of word-finding difficulties and impaired sentence repetition.

Based on these updated criteria, clinico-pathological studies have shown that $A D$ pathology often underlies lvPPA, whereas nfvPPA and svPPA are typically caused by frontotemporal lobar degeneration (FTLD) pathology (19-25). However, despite the application of international consensus clinical criteria, the prevalence of $A \beta$ pathology either measured at autopsy (19-26) or using in vivo biomarkers such as cerebrospinal fluid (CSF) analysis or positron emission tomography (PET) (27-38) - remained highly variable in single-center studies of PPA variants: $57-100 \%$ in lvPPA, 0-46\% in nfvPPA and $0-33 \%$ in svPPA. Given an estimated prevalence of 3.0/100,000 inhabitants for PPA (33), a multicenter approach is essential to overcome statistical power issues. We therefore performed an individual patient meta-analysis including 1,251 PPA patients from 36 dementia centers. The primary objective was to provide prevalence estimates of A $\beta$ pathology (determined at autopsy, CSF and/or PET) for each PPA variant. In secondary analyses, we evaluated relationships between $A \beta$ positivity and the main risk factors for $A \beta$ deposition, notably age and presence of an Apolipoprotein $E$ (ApoE) $\varepsilon 4$ allele. Furthermore, in a subset of patients with autopsy data available, we assessed the prevalence of neuropathological substrates in the different PPA variants.

\section{Patients and methods}

\subsection{Participating centers}

We searched the MEDLINE and Web of Science databases for biomarker (i.e. PET and/or CSF) or autopsy studies in PPA patients. The search terms were primary

This article is protected by copyright. All rights reserved. 
progressive aphasia or PPA combined with biomarkers, pathology, autopsy, neuropathology, cerebrospinal fluid, CSF, PET, PiB, Pittsburgh, florbetapir, AV-45, florbetaben, flutemetamol, amyloid, abeta, frontotemporal and Alzheimer's disease. 1,012 titles and abstracts were reviewed, resulting in 37 unique cohorts for which we contacted the study corresponding author to obtain primary data. In addition, we contacted principal investigators of dementia centers known to be involved in PPA/FTD research but did not (yet) publish a paper on the specific issue of $A \beta$ pathology in PPA. In total, we asked 42 study contact persons to provide participant-level data on $A \beta$ status, age, sex, education, handedness, APOE $\varepsilon 4$ status, Mini-Mental State Examination (MMSE) score, and Clinical Dementia Rating (CDR) scale score. Six centers declined or did not respond, leaving participant-level data from 36 cohorts for analysis (see eTable 1). We requested contributors to send both published and unpublished data. Informed consent was obtained from all patients or their assigned surrogate decision makers, and the institutional review boards for human research of the participating centers approved all studies.

\subsection{Data collection and operationalization}

Information on study procedures, extracted from the publication or provided by the study contact person, was used to create a common set of variables.

\section{Patients}

Patients had to fulfil core criteria for PPA (i.e. language impairment being the earliest and most prominent clinical feature and the principal cause of impaired activities of daily living at least during the first 2 years after disease onset) $(8,18)$. Patients were classified by contributing centers according to the PPA consensus criteria (18) as IvPPA, nfvPPA, svPPA, PPA-mixed (PPA-M; fulfils criteria for multiple PPA variants) or PPAunclassified (PPA-U; does not fulfil criteria for any specific variant despite meeting core criteria for PPA). Due to small sample sizes, we aggregated PPA-M and PPA-U into a

This article is protected by copyright. All rights reserved. 
single "PPA-M/U” group. Since current PPA consensus criteria were published in 2011 (18), we requested contributing centers to re-classify patients diagnosed before 2011 according to the current diagnostic framework by retrospectively reviewing patient charts, including clinical and imaging information (i.e. structural magnetic resonance imaging [MRI] and/or ${ }^{18} \mathrm{~F}$-fluorodeoxyglucose positron emission tomography [FDGPET]), excluding $A \beta$ biomarkers and autopsy results. All diagnoses were made locally using site specific clinical work-up. In line with the PPA consensus criteria [18], structural and functional imaging could be used to refine the clinical diagnosis. To minimize circularity biases, we emphasized that contributors should provide their working diagnosis prior to obtaining amyloid PET or lumbar puncture. However, since this is a retrospective study, there is no reliable measure to verify whether this was respected for all cases.

\section{PET and CSF procedures}

PET scans were dichotomized ( $\mathrm{A} \beta+$ or $\mathrm{A} \beta$-) using quantitative thresholds or visual reads according to the method used at the study site. Likewise, CSF measurements were dichotomized $(\mathrm{A} \beta+/ \mathrm{A} \beta-)$ using center-specific cut-offs. Detailed PET and CSF procedures for all participating cohorts are presented in eTable 2 and 3. When PET scanning was performed for clinical purposes, the PET readers were generally not blinded to the clinical diagnosis. In total, 93 patients had multiple measure of $\mathrm{A} \beta$ pathology available (62 PET + CSF, 19 PET + autopsy, 12 CSF + autopsy), yielding 92\% concordance between modalities. Patients were rated $A \beta+$ if at least one of the modalities revealed presence of $\mathrm{A} \beta$ positivity.

\section{Autopsy data}

Autopsy cases were assessed by certified neuropathologists following the National Institute on Aging-Alzheimer's Association (NIA-AA; (39)) or NIA-Reagan (40) 
guidelines for the neuropathologic assessment of AD. All centers provided a measure of amyloid pathology. In addition, some centers provided Braak stage and neuropathological diagnosis of $A D$. Patients were dichotomized $(A \beta+/ A \beta-)$ based on their Thal $A \beta$ plaque score (i.e. Thal phase $\geq 3$ ) and/or CERAD criteria (i.e. definite, probable or possible AD, indicating moderate to frequent neuritic plaques). In 10/13 autopsy studies (234/378 patients), neuropathological assessment also included screening for other pathologies, including tau pathologies (Pick's disease [PiD], corticobasal degeneration [CBD], progressive supranuclear palsy [PSP]; (41)), TAR DNA-binding protein 43 pathologies (TDP-43; type A, B or C); (42)), $\alpha$-synuclein (dementia with Lewy bodies [DLB]; (43)), cerebrovascular disease, argyrophilic grain disease, prions and FTLD-FUS. Some patients were analyzed prior to the discovery of TDP subtypes and were therefore coded TDP-unspecified (TDP-U). A $\beta$ pathology was considered "co-morbid" when combined with another full-blown pathology (FTLD-TDP, primary tauopathy) in the absence of semi-quantitative neuritic senile plaque density (CERAD score), and NFT severity/distribution (Braak stage) adding up to a "high" or "moderate” likelihood of AD.

\section{Clinical measures and genetic testing}

The Mini-Mental State Examination (MMSE) score (measure of global cognition) was available for 945 patients with PPA (76\%). Information on APOE genotype was available for 487 patients (39\%). None of the participating centers' cohorts were enriched for positive ApoE4 status. Age and gender were available for 1,167 (93\%) and 1,203 (96\%) patients, respectively.

\subsection{Statistical analyses}

We conducted a meta-analysis with individual participant data. Baseline characteristics were compared using analysis of variance, Fisher exact and Pearson's $\chi^{2}$ tests, where appropriate. Similar to previous meta-analyses $(36,44)$, generalized estimating equations 
(GEE, using SPSS software [IBM], version 23.0) models were used to estimate probabilities for $A \beta$ positivity. GEE were used since they allow analysis of binarycorrelated data, hence participant-level data from all cohorts can be modeled while simultaneously accounting for patients within cohorts. A logit link function for binary outcome with an exchangeable correlation structure was assumed to account for withinstudy correlation. Analyses were conducted using the total study population, unless specified otherwise. The main analysis was performed with diagnosis and age as independent variables, adjusted for center effects. Age as entered as a continuous measure centered at the median. We tested 2-way interactions between variables, and these terms were retained in the model if they were significant by the Wald statistical test. APOE $\varepsilon 4$ status was added to the model in secondary analysis of a subset of patients $(487 / 1,251)$. The slope for each PPA variant according to age were compared to those of probable (mostly amnestic-predominant) AD patients $(\mathrm{n}=1,359)$ and of cognitively normal individuals $(\mathrm{n}=2,914)$, derived from our previous meta-analyses (36) (44).

The degree of heterogeneity in prevalence of amyloid positivity across cohorts was assessed using the $\mathrm{I}^{2}$ statistic (generated by a random-effects meta-analysis in STATA (StataCorp) software version 14). An $\mathrm{I}^{2}$ statistic value greater than $50 \%$ indicates substantial heterogeneity (45). Significance level was set at two-sided $\alpha=0.05$. GraphPad Prism (GraphPad software) version 6.0 was used for the Figures.

\section{Results}

A total of 1,251 patients diagnosed with PPA (lvPPA [n=443], nfvPPA [n=333], svPPA [n=401], PPA-M/U [n=74]) and a measure of A $\beta$ pathology (CSF [n=600]), PET [n=366] or autopsy [n=378]); 93 cases with $\geq 2$ modalities) were included from 36 centers (Table 
1). Approximately a third of these cases $(425 / 1,251 ; 34 \%)$ were included in previous publications (eTable 1).

\section{Demographic and clinical data}

The mean age at $A \beta$ measurement was $67.3 \pm 8.1$ in the total PPA cohort (Table 1). Patients with svPPA were slightly younger than those with other PPA variants. Gender was equally distributed across all PPA variants, except in PPA-M/U in which females were underrepresented 30/74 (40.5\%). In all PPA variants, patients were on average highly educated (13.8 \pm 4.5 years). Consistent with the general population (non-righthandedness in $8-10 \%$ (46)), 68/809 (8.4\%) patients were left-handed or ambidextrous. Non-right-handedness was more prevalent in svPPA (10.8\%) than in nfvPPA (4.7\%; $\mathrm{p}<0.05)$. Prevalence of APOE $\varepsilon 4$ allele was higher in lvPPA (42.1\%) than in svPPA (26.3\%) and nfvPPA (20.2\%), and higher in PPA-M/U (37.2\%) than in nfvPPA. MMSE

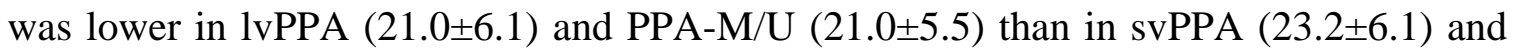
nfvPPA (24.0 \pm 5.7$)$.

\section{Prevalence of $A \beta$ positivity according to diagnosis}

About half (43.4\%) of all PPA patients were $A \beta$ positive. The prevalence of $A \beta$ positivity was greater in lvPPA (85.6\%) than in nfvPPA $(19.5 \%)$ and svPPA $(15.7 \%)(\mathrm{p}<0.001)$. 36/74 (48.6\%) of PPA-M/U were A $\beta+$.

\section{Prevalence of $A \beta$ positivity by modality}

Prevalence of $\mathrm{A} \beta$ positivity within PPA variants was consistent across all three modalities (all $\mathrm{p}>0.05$ ), except for a higher $\mathrm{A} \beta$ positivity in CSF than PET in svPPA $(\mathrm{p}<0.05)$ and a trend towards higher $\mathrm{A} \beta$ positivity in PET than autopsy in lvPPA ( $\mathrm{p}=0.09$, Figure 2). 93 patients had an $A \beta$ pathology measure derived from more than one modality, yielding a $92 \%$ concordance rate.

This article is protected by copyright. All rights reserved. 
Prevalence of $A \beta$ positivity according to age

The estimated prevalence of $A \beta$ positivity across the age span for variants of PPA is presented in Table 2. In the total sample, A $\beta$ positive PPA patients were older than $A \beta$ PPA patients $(68.4 \pm 7.8$ vs. $66.4 \pm 8.2, \mathrm{p}<0.001)$. Within PPA variants, $\mathrm{A} \beta+\mathrm{nfvPPA}$, svPPA and PPA-M/U patients were older than their $A \beta$ - counterparts patients $(70.8 \pm 8.3$ vs $68.2 \pm 8.3,69.0 \pm 6.5$ vs, $64.5 \pm 7.7,71.4 \pm 7.4$ vs $67.3 \pm 8.6$, all $\mathrm{p}<0.05)$. GEE analyses revealed that $A \beta$ positivity increased with age in nfvPPA and svPPA ( $\beta$ for change in prevalence per year \pm standard error: $0.05 \pm 0.02, \quad \mathrm{p}<0.01 ; 0.08 \pm 0.02, \quad \mathrm{p}<0.001$, respectively), but not in lvPPA $(\mathrm{p}=0.94)$ or $\mathrm{PPA}-\mathrm{M} / \mathrm{U}(\mathrm{p}=0.09$, Figure 1$)$. Of note, the slope for lvPPA patients closely resembled that of probable AD patients $(n=1,359)(36)$, while the slopes for svPPA and nfvPPA strongly overlapped with the slope for cognitively normal individuals ( $\mathrm{n}=2,914)$ (44) (Figure 1).

\section{Prevalence of $A \beta$ positivity according to $A P O E$}

Across PPA variants, APOE $\varepsilon 4$ carriers were more often amyloid- $\beta$ positive (58.0\%) than non-carriers (35.0\%, $\mathrm{p}<0.001)$. Within diagnostic groups, GEE analyses revealed main effects of APOE on prevalence of amyloid positivity in nfvPPA ( $\beta$ for difference in prevalence for carriers vs non-carriers \pm standard error: $1.22 \pm 0.55, \mathrm{p}<0.05)$, but not in $\operatorname{lvPPA}(\mathrm{p}=0.54)$, svPPA ( $\mathrm{p}=0.06)$ and PPA-M/U $(\mathrm{p}=0.30)$.

\section{Autopsy results in distinct PPA variants}

Autopsy results were available for 357 PPA patients (99 lvPPA, 109 nfvPPA, 106 svPPA and $43 \mathrm{PPA}-\mathrm{M} / \mathrm{U}$, Figure 3). Most patients with lvPPA had primary AD pathology (76\%), followed by FTLD TDP pathology (14\%, mostly type A) or FTLD tau pathology (5\%; See Figure 3). NfvPPA patients showed the most heterogeneous pathology across PPA variants. Most patients with nfvPPA had FTLD with primary tau pathology (64\%) -

This article is protected by copyright. All rights reserved. 
either corticobasal degeneration (CBD, 29\%), progressive supranuclear palsy (PSP, 17\%) or Pick's disease (PiD, 18\%) - followed by FTLD TDP pathology (24\%, mostly type A) or AD pathology (8\%). The vast majority of svPPA patients had TDP pathology (80\%; mostly type C [73\%]), with some patients exhibiting tau (11\%) or AD (5\%) pathology. PPA-M/U was divided between FTLD tau (35\%), FTLD TDP (21\%) and AD (42\%) pathologies. The presence of FTLD tau, FTLD TDP-C and AD pathology was associated with particular PPA phenotypes: 77/78 (99\%) of FTLD TDP-C patients had a clinical diagnosis of svPPA, 75/107 (70\%) AD+ cases had lvPPA and 70/102 (69\%) FTLD tau cases had nfvPPA. In contrast, FTLD TDP type A pathology was associated with heterogeneous language profiles (among 35 TDP-A+ cases, 10 were lvPPA, 16 nfvPPA, 1 svPPA and 8 PPA-M/U). A $\beta$ pathology was often co-morbid (rather than the causative etiology) to primary tau/TDP pathology in nfvPPA (10/19 [53\%] of A $\beta+$ cases) and svPPA (5/10 [50\%]), but not in lvPPA (5/79 [6\%]) and PPA-M/U (4/22 [18\%]). Some cases of PPA exhibited atypical pathologies such as Creutzfeld-Jackob disease (CJD; 2/357), dementia with Lewy bodies (DLB; 9/357), argyrophilic grain disease (AGD; 1/357), vascular dementia (VaD; 1/357), FTLD-FUS (1/357) and globular glial tauopathy (GGT; 2/357).

Assessment of study-related heterogeneity

According to the $\mathrm{I}^{2}$ statistic, there was no substantial heterogeneity in the prevalence of $A \beta$ positivity between centers for any of the diagnostic groups (lvPPA [29.6\%, $\left.X^{2}=42,64\right]$, nfvPPA [28.9\%, $\left.X^{2}=37.96\right]$, svPPA [13.5\%, $\left.X^{2}=33.53\right]$, PPA-M/U [2.2\%, $\left.\mathrm{X}^{2}=12.27\right]$, all $\left.\mathrm{p}>0.05\right)$.

\section{Discussion}

This article is protected by copyright. All rights reserved. 
In this multicenter study involving 1,251 patients diagnosed with PPA and a measure of $A \beta$ pathology (CSF, PET or autopsy), we found that $A \beta$ positivity is more prevalent in lvPPA (86\%) than in nfvPPA (20\%) or svPPA (16\%). The prevalence of A $\beta$ positivity increased with advancing age in nfvPPA and svPPA (similar to cognitively normal subjects (44)), but not in lvPPA (in line with probable AD patients (36)). Furthermore, svPPA and nfvPPA patients carrying a major risk allele for sporadic AD (i.e. APOE $\varepsilon 4$ ) were more often $A \beta$ positive than non-carriers. These results demonstrate the utility of the new classification system (18) to predict underlying pathology of PPA in various clinical settings and suggest that age and APOE genotype should be taken into account when interpreting $A \beta$ biomarkers in PPA patients.

In previous individual studies, the prevalence of $\mathrm{A} \beta$ pathology detected using in vivo biomarkers or neuropathological examination in patients with distinct variants of PPA differed widely: between $57-100 \%$ in lvPPA, $0-46 \%$ in nfvPPA and 0-33\% in svPPA (1926, 38). This meta-analysis using individual participant data from 36 centers showed that $\mathrm{A} \beta$ pathology was present in the vast majority of patients with lvPPA (86\%) and in a minority of nfvPPA (20\%) and svPPA (16\%) cases. Furthermore, autopsy data from 357 PPA patients revealed that AD pathology was indeed the major driving force in lvPPA (76\%), while FTLD TDP-43 (80\%, mostly type C) and FTLD tau (64\%) pathology where most prevalent in svPPA and nfvPPA, respectively. These findings are consistent with the notion of selective vulnerability of neural networks to specific proteinopathies, with $\mathrm{AD}$ pathology having a tropism towards posterior temporoparietal brain regions, tau pathology towards fronto-striatal networks and TDP-C pathology towards the temporal pole $(19,47,48)$. When these pathologies demonstrate lateralization towards the language-dominant hemisphere, this may result in distinct variants of PPA (lvPPA, nfvPPA or svPPA, respectively) The mechanisms underlying lateralization of pathology in PPA remain a mystery. It has been suggested that left-handedness or developmental 
learning disabilities (e.g. dyslexia) may increase vulnerability of the language-dominant hemisphere to neurodegenerative disorders $(49,50)$. In this multicenter study, the proportion of non-righthandedness was similar among PPA variants and consistent with the general population (46), and there were no data available for learning disabilities. However, handedness data was only available in 25/36 centers for 809/1,251 patients (65\%), and was not evaluated thoroughly using a handedness questionnaire, hence forced right-handedness may be a potential confounder (51). Genome-wide association studies (GWAS) might shed light on potential contributors to dominant-hemisphere vulnerability in PPA. Similarly, a recent genome wide association study in posterior cortical atrophy (the "visual variant of $\mathrm{AD}$ ") revealed associations with genes involved in neurodevelopment of the visual system and retinal degeneration (52).

This study highlights that caution is needed in interpreting the significance of amyloid biomarkers in PPA. Several findings suggest that $A \beta$ pathology may be an age-related process in svPPA and nfvPPA, co-morbid to primary FTLD pathology (i.e. TDP-43 or $3 R / 4 R$ tau). First, there is a strong increase of $A \beta$-positivity in clinical syndromes mostly associated with non-AD pathologies (i.e. nfvPPA and svPPA) with the presence of two main risk factors for sporadic AD (i.e. aging and APOE $\varepsilon 4$ ). Second, the slopes of increased $A \beta$-positivity according to age in nfvPPA and svPPA (Figure 1) bear strong resemblance with that of cognitively normal elderly (44). Finally, our autopsy results showed that more than half of $A \beta+$ nfvPPA/svPPA patients exhibit concomitant FTLD pathology, compared to only $6 \%$ of $\mathrm{A} \beta+\operatorname{lvPPA}$ patients (See Figure 3 ), which is consistent with several case reports showing that positive $A \beta$ biomarkers do not necessarily indicate that the clinical syndrome is primarily driven by AD pathology (5355). Previous reports have shown that APOE4 allele was a risk factor for amyloidpositivity but not for neuropathological diagnosis of AD in PPA patients $(12,23,25)$. This has potential clinical ramifications, as the increased a priori likelihood of detecting 
(comorbid) $\mathrm{A} \beta$ pathology in older patients and/or APOE $\varepsilon 4$ carriers should be taken into account when interpreting $A \beta$ biomarkers in patients with PPA. For example, in older patients with a clear clinical profile of nfvPPA or svPPA, a positive amyloid PET should be interpreted with caution, as amyloid PET has lower positive predictive value for AD neuropathology in such patients $(55,56)$. On the other hand, it is possible that co-morbid age-related $A \beta$ pathology may not be an innocent bystander, as recent data suggests that it is associated with worse cognition and greater probability of clinical expression of different dementia syndromes $(36,57,58)$.

Apart from the possibility of dual pathologies, there are several other explanations for diverging biomarker results in PPA (e.g. A $\beta$ - lvPPA or $A \beta+$ nfvPPA/svPPA). First, the imperfect clinico-pathological correlations in PPA may reflect the incomplete tropism of pathogenic proteins for specific brain networks, as proteinopathies sometimes arise in nodes outside their typical "signature" networks $(47,48)$. Recent studies showed that $\mathrm{A} \beta+$ and $\mathrm{A} \beta$ - lvPPA patients showed differential clinical features and hypometabolic deficits at $\left[{ }^{18} \mathrm{~F}\right]$ FDG-PET, suggesting that deeper clinical/anatomical phenotyping of lvPPA patients could help better predicting the underlying pathology (35, 59, 60). Second, the in vivo biomarkers could possibly have provided false positive or false negative results. This is likely only a partial explanation, however, as A $\beta$ PET and CSF assessments correspond well with neuropathological examination $(61,62)$, and the three modalities provided similar results in this study. Finally, imperfect clinico-pathological correlations could be attributed to clinical misdiagnosis and/or variable interpretation of the clinical criteria. The implementation of standardized tests to assess key language features of PPA (e.g. repetition, agrammatism, comprehension) might help increase interrater reliability of PPA diagnosis. For instance, patients with lvPPA may successfully repeat short/simple sentences (and hence be diagnosed with PPA-U), yet would show impairment at more complex repetition tasks (26, 34, 63). 
The consensus criteria for PPA captured the vast majority of language profiles of patients in this study. Only $5.9 \%$ of PPA could not be classified into one of the three variants, either because they had unclassifiable $(n=40)$ or mixed $(n=34)$ phenotypes. The unclassifiable phenotype primarily included patients with word retrieval and naming deficits who did not fit a diagnosis of lvPPA due to not meeting the core criterion of abnormal repetition. Most patients with a mixed phenotype exhibited core features of multiple PPA variants, for instance a combination of agrammatism and word comprehension deficits. Likewise, some patients who fit the semantic or agrammatic classification also fulfilled criteria for lvPPA. The prevalence of mixed/unclassifiable phenotypes is consistent with most large cohorts published to date (19, 22-24, 33, 38), but lower than others (64-67). The heterogeneous pathologies found at autopsy of PPA-M/U suggest that the ambiguities in the current PPA classification cannot be easily resolved through the addition of a fourth clinical variant. Of note, patients with a pure phenotype of primary progressive apraxia of speech (68) were not included in the study if they did not fulfill core criteria for PPA. Many included nfvPPA patients were reported to have predominant apraxia of speech, yet still exhibited language impairments (agrammatism, writing/reading difficulties) consistent with PPA. Finally, MMSE scores were lower in

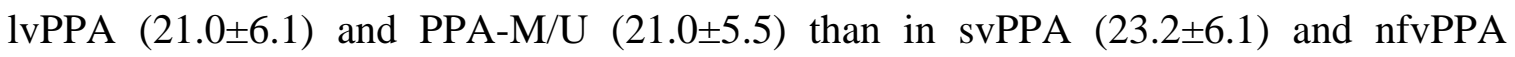

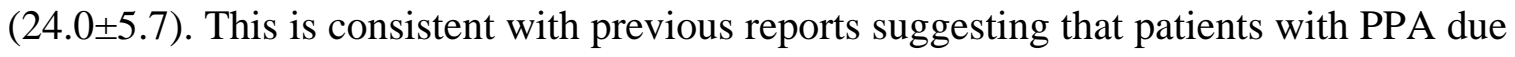
to $\mathrm{AD}$ have greater memory, visuospatial and executive impairment than other PPA variants (69-72).

Strengths of this study include the large sample size $(n=1,251)$ from 36 centers, and the inclusion of various measures of A $\beta$ pathology (CSF, PET and autopsy). Our study also has limitations. First, due to the retrospective nature of the study, there remains an inherent risk for circularity biases, with biomarker results influencing diagnostic 
classification (or vice versa) due to assumptions regarding clinico-pathological correlations. Although we emphasized that co-investigators provide patients' working diagnosis prior to the biomarker study (i.e. agnostic to $A \beta$ status), we cannot reliably confirm that this was respected in all centers. Likewise, as some scans were performed on a clinical basis - hence readers were not blinded to the clinical diagnosis - we cannot exclude that knowledge of clinical diagnosis has influenced PET visual interpretation in some borderline cases. Prospective studies are needed to mitigate these potential circularity biases. Second, differences in clinical work-up across centers - with variable use of neuropsychological assessment, speech/language pathology, and structural and functional imaging - likely had an influence on patients' classification. Uniformly applied, research-level phenotyping of PPA patients would likely have resulted in stronger clinico-pathological correlation $(19,38)$. On the other hand, our study was able to assess the current classification system across a diverse spectrum of clinical settings. Third, acquisition and interpretation methods for PET and CSF were not harmonized across cohorts (e.g. different PET tracers, CSF analytical steps, neuropathological procedures). We addressed this using center or method specific cut-points and corrected for center effects. Importantly, $\mathrm{I}^{2}$ statistics did not reveal significant study-related heterogeneity between center. Furthermore, post hoc analyses showed no significant differences across modalities. Fourth, when interpreting this study, some sample characteristics should be taken into account. For example, most patients visited tertiary referral centers and patients were highly educated (13.8 years on average). Finally, since the majority of PPA research focused on the FTLD spectrum, APOE genotype information was only available in $\sim 40 \%$ of the sample.

In conclusion, this multicenter study helps refining our understanding of clinicopathological correlations in PPA. In future studies, further investigations of clinical, structural/functional imaging and genetic features of PPA are needed to increase our 
knowledge of PPA pathogenesis. This will improve accuracy of the PPA diagnosis and the identification of the underlying etiology, which will lead to more accurate and efficient participant inclusion in clinical trials with disease modifying agents tailored to reduce cerebral A $\beta$, tau and/or TDP-43 pathology. Furthermore, the field would benefit from a prospective multicenter trial assessing the potential benefit of cholinesterase inhibitors in lvPPA and other A $\beta+$ PPA variants. As with most rare disorders (prevalence: 3.0/100,000), PPA will benefit from tight collaboration between researchers worldwide to obtain sufficient sample size.

This article is protected by copyright. All rights reserved. 


\section{FIGURE LEGENDS}

Figure 1. Prevalence of amyloid- $\beta$ positivity in primary progressive aphasia variants

Prevalence estimate amyloid- $\beta$ positivity based on GEE analyses.

Note: data for normal controls and typical AD dementia come from the Amyloid PET Study Group $(36,44)$

Figure 2. Prevalence of A $\beta$ pathology in PPA variants by modality

93 patients had multiple measure of A $\beta$ pathology available (62 PET + CSF, 19 PET + autopsy, 12 CSF + autopsy), yielding 92\% concordance between modalities.

Figure 3. Autopsy results

Panel A: Pie charts showing the respective prevalence of amyloid, tau, TDP and other pathologies in PPA.

Panel B: breakdown of the different pathologies for each PPA variant.

Abbreviations: AGD: argyrophilic grain disease; CBD: corticobasal degeneration; CJD: Creutzfeld-Jackob disease; DLB: dementia with Lewy bodies; GGT: globular glial tauopathy; lvPPA: logopenic variant PPA; nfvPPA; non-fluent variant of PPA; PiD: Pick's disease; PPA-M/U: mixed/unclassifiable PPA; PSP: progressive supranuclear palsy; svPPA: semantic variant of PPA; TDP: TAR DNA-binding protein 43; TDP-U: TDP unclassified; VaD: vascular dementia. 


\title{
Acknowledgments
}

We wish to thank patients and families for their dedication to research. This work was funded by a Vanier Graduate Scholarship of the Canadian Institutes of Health Research.

\begin{abstract}
Author contributions
DB and RO designed the study and coordinated data sharing. DB, CG, PJV and RO performed data analysis. DB, MLGT, GDR, CG, RJrLG and RO drafted the manuscript. All authors contributed to the acquisition of data as well as data analysis, provided intellectual input to the manuscript and approved its final version.
\end{abstract}

\section{Potential Conflicts of interest}

Nothing to report.

This article is protected by copyright. All rights reserved. 


\section{References}

1. Mesulam MM, Rogalski EJ, Wieneke C, Hurley RS, Geula C, Bigio EH, et al. Primary progressive aphasia and the evolving neurology of the language network. Nat Rev Neurol. 2014;10(10):554-69.

2. Mesulam MM. Slowly progressive aphasia without generalized dementia. Ann Neurol. 1982;11(6):592-8.

3. Neary D, Snowden JS, Gustafson L, Passant U, Stuss D, Black S, et al. Frontotemporal lobar degeneration: a consensus on clinical diagnostic criteria. Neurology. 1998;51(6):1546-54.

4. Greene JD, Patterson K, Xuereb J, Hodges JR. Alzheimer disease and nonfluent progressive aphasia. Arch Neurol. 1996;53(10):1072-8.

5. Kempler D, Metter EJ, Riege WH, Jackson CA, Benson DF, Hanson WR. Slowly progressive aphasia: three cases with language, memory, CT and PET data. J Neurol Neurosurg Psychiatry. 1990;53(11):987-93.

6. Pogacar S, Williams RS. Alzheimer's disease presenting as slowly progressive aphasia. R I Med J. 1984;67(4):181-5.

7. Mesulam MM. Primary progressive aphasia--differentiation from Alzheimer's disease. Ann Neurol. 1987;22(4):533-4.

8. Mesulam MM. Primary progressive aphasia. Ann Neurol. 2001;49(4):425-32.

9. Kertesz A, Hudson L, Mackenzie IR, Munoz DG. The pathology and nosology of primary progressive aphasia. Neurology. 1994;44(11):2065-72.

10. Gorno-Tempini ML, Dronkers NF, Rankin KP, Ogar JM, Phengrasamy L, Rosen HJ, et al. Cognition and anatomy in three variants of primary progressive aphasia. Ann Neurol. 2004;55(3):335-46.

11. Rabinovici GD, Jagust WJ, Furst AJ, Ogar JM, Racine CA, Mormino EC, et al. Abeta amyloid and glucose metabolism in three variants of primary progressive aphasia. Ann Neurol. 2008;64(4):388-401.

12. Mesulam M, Wicklund A, Johnson N, Rogalski E, Leger GC, Rademaker A, et al. Alzheimer and frontotemporal pathology in subsets of primary progressive aphasia. Ann Neurol. 2008;63(6):709-19.

13. Hu WT, McMillan C, Libon D, Leight S, Forman M, Lee VM, et al. Multimodal predictors for Alzheimer disease in nonfluent primary progressive aphasia. Neurology. 2010;75(7):595-602.

14. Knibb JA, Xuereb JH, Patterson K, Hodges JR. Clinical and pathological characterization of progressive aphasia. Ann Neurol. 2006;59(1):156-65.

15. Alladi S, Xuereb J, Bak T, Nestor P, Knibb J, Patterson K, et al. Focal cortical presentations of Alzheimer's disease. Brain. 2007;130(Pt 10):2636-45.

This article is protected by copyright. All rights reserved. 
16. Josephs KA, Whitwell JL, Duffy JR, Vanvoorst WA, Strand EA, Hu WT, et al. Progressive aphasia secondary to Alzheimer disease vs FTLD pathology. Neurology. 2008;70(1):25-34.

17. Grossman M. Primary progressive aphasia: clinicopathological correlations. Nat Rev Neurol. 2010;6(2):88-97.

18. Gorno-Tempini ML, Hillis AE, Weintraub S, Kertesz A, Mendez M, Cappa SF, et al. Classification of primary progressive aphasia and its variants. Neurology. 2011;76(11):1006-14.

19. Spinelli EG, Mandelli ML, Miller ZA, Santos-Santos MA, Wilson SM, Agosta F, et al. Typical and atypical pathology in primary progressive aphasia variants. Ann Neurol. 2017.

20. Rohrer JD, Lashley T, Schott JM, Warren JE, Mead S, Isaacs AM, et al. Clinical and neuroanatomical signatures of tissue pathology in frontotemporal lobar degeneration. Brain. 2011;134(Pt 9):2565-81.

21. Rohrer JD, Rossor MN, Warren JD. Alzheimer's pathology in primary progressive aphasia. Neurobiol Aging. 2012;33(4):744-52.

22. Chare L, Hodges JR, Leyton CE, McGinley C, Tan RH, Kril JJ, et al. New criteria for frontotemporal dementia syndromes: clinical and pathological diagnostic implications. J Neurol Neurosurg Psychiatry. 2014;85(8):865-70.

23. Mesulam MM, Weintraub S, Rogalski EJ, Wieneke C, Geula C, Bigio EH. Asymmetry and heterogeneity of Alzheimer's and frontotemporal pathology in primary progressive aphasia. Brain. 2014;137(Pt 4):1176-92.

24. Harris JM, Gall C, Thompson JC, Richardson AM, Neary D, du Plessis D, et al. Classification and pathology of primary progressive aphasia. Neurology. 2013;81(21):1832-9.

25. Rogalski E, Sridhar J, Rader B, Martersteck A, Chen K, Cobia D, et al. Aphasic variant of Alzheimer disease: Clinical, anatomic, and genetic features. Neurology. 2016;87(13):1337-43.

26. Giannini LAA, Irwin DJ, McMillan CT, Ash S, Rascovsky K, Wolk DA, et al. Clinical marker for Alzheimer disease pathology in logopenic primary progressive aphasia. Neurology. 2017.

27. Leyton CE, Villemagne VL, Savage S, Pike KE, Ballard KJ, Piguet O, et al. Subtypes of progressive aphasia: application of the International Consensus Criteria and validation using beta-amyloid imaging. Brain. 2011;134(Pt 10):3030-43.

28. Josephs KA, Duffy JR, Strand EA, Machulda MM, Senjem ML, Lowe VJ, et al. APOE epsilon4 influences beta-amyloid deposition in primary progressive aphasia and speech apraxia. Alzheimers Dement. 2014;10(6):630-6.

29. Ikeda M, Tashiro Y, Takai E, Kurose S, Fugami N, Tsuda K, et al. CSF levels of Abeta1-38/Abeta1-40/Abeta1-42 and (11)C PiB-PET studies in three clinical variants of primary progressive aphasia and Alzheimer's disease. Amyloid. 2014;21(4):238-45. 
30. Santangelo R, Coppi E, Ferrari L, Bernasconi MP, Pinto P, Passerini G, et al. Cerebrospinal fluid biomarkers can play a pivotal role in the diagnostic work up of primary progressive aphasia. J Alzheimers Dis. 2015;43(4):1429-40.

31. Gil-Navarro S, Llado A, Rami L, Castellvi M, Bosch B, Bargallo N, et al. Neuroimaging and biochemical markers in the three variants of primary progressive aphasia. Dement Geriatr Cogn Disord. 2013;35(1-2):106-17.

32. Kas A, Uspenskaya O, Lamari F, de Souza LC, Habert MO, Dubois B, et al. Distinct brain perfusion pattern associated with CSF biomarkers profile in primary progressive aphasia. J Neurol Neurosurg Psychiatry. 2012;83(7):695-8.

33. Magnin E, Demonet JF, Wallon D, Dumurgier J, Troussiere AC, Jager A, et al. Primary Progressive Aphasia in the Network of French Alzheimer Plan Memory Centers. J Alzheimers Dis. 2016;54(4):1459-71.

34. Louwersheimer E, Keulen MA, Steenwijk MD, Wattjes MP, Jiskoot LC, Vrenken $\mathrm{H}$, et al. Heterogeneous Language Profiles in Patients with Primary Progressive Aphasia due to Alzheimer's Disease. J Alzheimers Dis. 2016;51(2):581-90.

35. Matias-Guiu JA, Cabrera-Martin MN, Moreno-Ramos T, Valles-Salgado M, Fernandez-Matarrubia M, Carreras JL, et al. Amyloid and FDG-PET study of logopenic primary progressive aphasia: evidence for the existence of two subtypes. J Neurol. 2015;262(6):1463-72.

36. Ossenkoppele R, Jansen WJ, Rabinovici GD, Knol DL, van der Flier WM, van Berckel BN, et al. Prevalence of amyloid PET positivity in dementia syndromes: a metaanalysis. JAMA. 2015;313(19):1939-49.

37. Patricio CM, Gabriela C, Julieta RM, Marcos FS, Federico N, Griselda R, et al. Concordance between 11C-PIB-PET and clinical diagnosis in a memory clinic. Am J Alzheimers Dis Other Demen. 2015;30(6):599-606.

38. Santos-Santos MA, Rabinovici GD, Iaccarino L, Ayakta N, Tammewar G, Lobach I, et al. Rates of Amyloid Imaging Positivity in Patients With Primary Progressive Aphasia. JAMA Neurol. 2018.

39. Hyman BT, Phelps CH, Beach TG, Bigio EH, Cairns NJ, Carrillo MC, et al. National Institute on Aging-Alzheimer's Association guidelines for the neuropathologic assessment of Alzheimer's disease. Alzheimers Dement. 2012;8(1):1-13.

40. Hyman BT, Trojanowski JQ. Consensus recommendations for the postmortem diagnosis of Alzheimer disease from the National Institute on Aging and the Reagan Institute Working Group on diagnostic criteria for the neuropathological assessment of Alzheimer disease. J Neuropathol Exp Neurol. 1997;56(10):1095-7.

41. McKhann GM, Albert MS, Grossman M, Miller B, Dickson D, Trojanowski JQ, et al. Clinical and pathological diagnosis of frontotemporal dementia: report of the Work Group on Frontotemporal Dementia and Pick's Disease. Arch Neurol. 2001;58(11):18039. 
42. Mackenzie IR, Neumann M, Baborie A, Sampathu DM, Du Plessis D, Jaros E, et al. A harmonized classification system for FTLD-TDP pathology. Acta Neuropathol. 2011;122(1):111-3.

43. McKeith IG, Galasko D, Kosaka K, Perry EK, Dickson DW, Hansen LA, et al. Consensus guidelines for the clinical and pathologic diagnosis of dementia with Lewy bodies (DLB): report of the consortium on DLB international workshop. Neurology. 1996;47(5):1113-24.

44. Jansen WJ, Ossenkoppele R, Knol DL, Tijms BM, Scheltens P, Verhey FR, et al. Prevalence of cerebral amyloid pathology in persons without dementia: a meta-analysis. JAMA. 2015;313(19):1924-38.

45. Higgins JP, Thompson SG. Quantifying heterogeneity in a meta-analysis. Stat Med. 2002;21(11):1539-58.

46. Perelle IB, Ehrman L. An international study of human handedness: the data. Behav Genet. 1994;24(3):217-27.

47. Seeley WW. Mapping Neurodegenerative Disease Onset and Progression. Cold Spring Harb Perspect Biol. 2017.

48. Warren JD, Rohrer JD, Schott JM, Fox NC, Hardy J, Rossor MN. Molecular nexopathies: a new paradigm of neurodegenerative disease. Trends Neurosci. 2013;36(10):561-9.

49. Miller ZA, Mandelli ML, Rankin KP, Henry ML, Babiak MC, Frazier DT, et al. Handedness and language learning disability differentially distribute in progressive aphasia variants. Brain. 2013;136(Pt 11):3461-73.

50. Miller ZA, Hinkley LB, Herman A, Honma S, Findlay A, Block N, et al. Anomalous functional language lateralization in semantic variant PPA. Neurology. 2015;84(2):204-6.

51. Ellis SJ, Ellis PJ, Marshall E, Windridge C, Jones S. Is forced dextrality an explanation for the fall in the prevalence of sinistrality with age? A study in northern England. J Epidemiol Community Health. 1998;52(1):41-4.

52. Schott JM, Crutch SJ, Carrasquillo MM, Uphill J, Shakespeare TJ, Ryan NS, et al. Genetic risk factors for the posterior cortical atrophy variant of Alzheimer's disease. Alzheimers Dement. 2016;12(8):862-71.

53. Caso F, Gesierich B, Henry M, Sidhu M, LaMarre A, Babiak M, et al. Nonfluent/agrammatic PPA with in-vivo cortical amyloidosis and Pick's disease pathology. Behav Neurol. 2013;26(1-2):95-106.

54. Naasan G, Rabinovici GD, Ghosh P, Elofson JD, Miller BL, Coppola G, et al. Amyloid in dementia associated with familial FTLD: not an innocent bystander. Neurocase. 2016;22(1):76-83.

55. Mesulam MM, Dickerson BC, Sherman JC, Hochberg D, Gonzalez RG, Johnson KA, et al. Case 1-2017. A 70-Year-Old Woman with Gradually Progressive Loss of Language. N Engl J Med. 2017;376(2):158-67. 
56. Bergeron D, Ossenkoppele R, Jr Laforce R. Evidence-based Interpretation of Amyloid-beta PET Results: A Clinician's Tool. Alzheimer Dis Assoc Disord. 2018. 57. James BD, Wilson RS, Boyle PA, Trojanowski JQ, Bennett DA, Schneider JA. TDP-43 stage, mixed pathologies, and clinical Alzheimer's-type dementia. Brain. 2016;139(11):2983-93.

58. Jansen WJ, Ossenkoppele R, Tijms BM, Fagan AM, Hansson O, Klunk WE, et al. Association of Cerebral Amyloid-beta Aggregation With Cognitive Functioning in Persons Without Dementia. JAMA Psychiatry. 2018;75(1):84-95.

59. Whitwell JL, Duffy JR, Strand EA, Machulda MM, Senjem ML, Schwarz CG, et al. Clinical and neuroimaging biomarkers of amyloid-negative logopenic primary progressive aphasia. Brain Lang. 2015;142:45-53.

60. Leyton CE, Hodges JR, McLean CA, Kril JJ, Piguet O, Ballard KJ. Is the logopenic-variant of primary progressive aphasia a unitary disorder? Cortex. 2015;67:122-33.

61. Tapiola T, Alafuzoff I, Herukka SK, Parkkinen L, Hartikainen P, Soininen H, et al. Cerebrospinal fluid \{beta\}-amyloid 42 and tau proteins as biomarkers of Alzheimertype pathologic changes in the brain. Arch Neurol. 2009;66(3):382-9.

62. Clark CM, Pontecorvo MJ, Beach TG, Bedell BJ, Coleman RE, Doraiswamy PM, et al. Cerebral PET with florbetapir compared with neuropathology at autopsy for detection of neuritic amyloid-beta plaques: a prospective cohort study. Lancet Neurol. 2012;11(8):669-78.

63. Leyton CE, Savage S, Irish M, Schubert S, Piguet O, Ballard KJ, et al. Verbal repetition in primary progressive aphasia and Alzheimer's disease. J Alzheimers Dis. 2014;41(2):575-85.

64. Whitwell JL, Weigand SD, Duffy JR, Strand EA, Machulda MM, Senjem ML, et al. Clinical and MRI models predicting amyloid deposition in progressive aphasia and apraxia of speech. Neuroimage Clin. 2016;11:90-8.

65. Sajjadi SA, Patterson K, Nestor PJ. Logopenic, mixed, or Alzheimer-related aphasia? Neurology. 2014;82(13):1127-31.

66. Wicklund MR, Duffy JR, Strand EA, Machulda MM, Whitwell JL, Josephs KA. Quantitative application of the primary progressive aphasia consensus criteria. Neurology. 2014;82(13):1119-26.

67. Josephs KA, Duffy JR, Strand EA, Machulda MM, Vemuri P, Senjem ML, et al. Progranulin-associated PiB-negative logopenic primary progressive aphasia. J Neurol. 2014;261(3):604-14.

68. Josephs KA, Duffy JR, Strand EA, Machulda MM, Senjem ML, Master AV, et al. Characterizing a neurodegenerative syndrome: primary progressive apraxia of speech. Brain. 2012;135(Pt 5):1522-36. 
69. Watson CL, Possin K, Allen IE, Hubbard HI, Meyer M, Welch AE, et al. Visuospatial Functioning in the Primary Progressive Aphasias. J Int Neuropsychol Soc. 2017:1-10.

70. Butts AM, Machulda MM, Duffy JR, Strand EA, Whitwell JL, Josephs KA. Neuropsychological Profiles Differ among the Three Variants of Primary Progressive Aphasia. J Int Neuropsychol Soc. 2015;21(6):429-35.

71. Leyton CE, Hsieh S, Mioshi E, Hodges JR. Cognitive decline in logopenic aphasia: more than losing words. Neurology. 2013;80(10):897-903.

72. Macoir J, Lavoie M, Laforce R, Jr., Brambati SM, Wilson MA. Dysexecutive Symptoms in Primary Progressive Aphasia: Beyond Diagnostic Criteria. J Geriatr Psychiatry Neurol. 2017;30(3):151-61.

This article is protected by copyright. All rights reserved. 


\section{University Library}

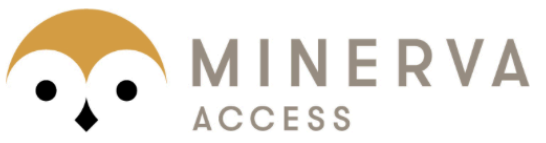

\section{A gateway to Melbourne's research publications}

Minerva Access is the Institutional Repository of The University of Melbourne

\section{Author/s:}

Bergeron, D;Gorno-Tempini, ML;Rabinovici, GD;Santos-Santos, MA;Seeley, W;Miller, BL;Pijnenburg, Y;Keulen, MA;Groot, C;van Berckel, BNM;van der Flier, WM;Scheltens, P;Rohrer, JD;Warren, JD;Schott, JM;Fox, NC;Sanchez-Valle, R;Grau-Rivera, O;Gelpi, E;Seelaar, H;Papma, JM;van Swieten, JC;Hodges, JR;Leyton, CE;Piguet, O;Rogalski, EJ;Mesulam, MM;Koric, L;Nora, K;Pariente, J;Dickerson, B;Mackenzie, IR;Hsiung, GYR;Belliard, S;Irwin, DJ;Wolk, DA;Grossman, M;Jones, M;Harris, J;Mann, D;Snowden, JS;Chrem-Mendez, P;Calandri, IL;Amengual, AA;Miguet-Alfonsi, C;Magnin, E;Magnani, G;Santangelo, R;Deramecourt, V;Pasquier, F;Mattsson, N;Nilsson, C;Hansson, O;Keith, J;Masellis, M;Black, SE;Matias-Guiu, JA;Cabrera-Martin, M-N;Paquet, C;Dumurgier, J;Teichmann, M;Sarazin, M;Bottlaender, M;Dubois, B;Rowe, CC;Villemagne, VL;Vandenberghe, R;Granadillo, E;Teng, E;Mendez, M;Meyer, PT;Frings, L;Lleo, A;Blesa, R;Fortea, J;Seo, SW;Diehl-Schmid, J;Grimmer, T;Frederiksen, KS;Sanchez-Juan, P;Chetelat, G;Jansen, W;Bouchard, RW;Laforce, R;Visser, PJ;Ossenkoppele, R

Title:

Prevalence of amyloid-beta pathology in distinct variants of primary progressive aphasia

Date:

2018-11-01

\section{Citation:}

Bergeron, D., Gorno-Tempini, M. L., Rabinovici, G. D., Santos-Santos, M. A., Seeley, W., Miller, B. L., Pijnenburg, Y., Keulen, M. A., Groot, C., van Berckel, B. N. M., van der Flier, W. M., Scheltens, P., Rohrer, J. D., Warren, J. D., Schott, J. M., Fox, N. C., Sanchez-Valle, R., Grau-Rivera, O., Gelpi, E. ,... Ossenkoppele, R. (2018). Prevalence of amyloid-beta pathology in distinct variants of primary progressive aphasia. ANNALS OF NEUROLOGY, 84 (5), pp.729-740. https://doi.org/10.1002/ana.25333.

Persistent Link:

http://hdl.handle.net/11343/284524 